\title{
Influence of Carrier and Gear Manufacturing Errors on the Static Load Sharing Behavior of Planetary Gear Sets*
}

\author{
Ajit BODAS $^{* *}$ and Ahmet KAHRAMAN***
}

\begin{abstract}
In this paper, a state-of-the-art contact mechanics model of a planetary gear set is employed to study the effect of a number of manufacturing and assembly related carrier and gear errors on the load sharing amongst the planets. Three different groups of errors are considered: (i) time-invariant, assembly-independent errors such as carrier planet pinhole position errors, (ii) time-invariant, assembly-dependent errors such as planet tooth thickness errors, and (iii) time-varying, assembly-dependent errors such as gear run-out errors. With such errors present, planet load sharing characteristics of an $n$-planet system $(n=3$ to 6$)$ is investigated for different piloting configurations under quasi-static conditions. Load sharing behavior as a function of key manufacturing errors is quantified and design guidelines are proposed for better planet load sharing behavior.
\end{abstract}

Key Words: Planetary Gear Sets, Planet Load Sharing

\section{Introduction}

In an $n$-planet planetary gear system, the input torque applied should theoretically be shared equally by each planet, i.e. each planet should carry $1 / n$ of the total input torque. When certain manufacturing errors and assembly variations are present, such an ideal load sharing condition is often not possible. Each of these errors and variations influence the position of the planet tooth flanks that mesh with the sun gear and the internal gear. If the errors or variations are such that a certain planet is pushed ahead of the rest in its relative tangential position within the carrier, it is likely that it will carry more load than the others.

As a large number of manufacturing errors can influence the planet load sharing characteristics ${ }^{(1)}$, they can be classified in three distinct groups. The first of these groups include time-invariant, assembly independent errors. Such errors maintain their value as the planet carrier rotates. Examples of these type errors include the planet pinhole position errors and pinhole diameter errors. The second group is formed by time-invariant, assembly dependent errors. These errors, once the planetary gear set is assembled, maintain their value as the carrier rotates. Planet tooth

* Received 20th October, 2003 (No. 03-5125)

** MIME Department, The University of Toledo, Toledo, Ohio 43617, U.S.A.

*** Department of Mechanical Engineering, The Ohio State University, 206 W. 18th Avenue, Columbus, Ohio 43210, U.S.A. E-mail: kahraman.1@osu.edu thickness errors, planet bore diameter errors, planet bearing needle diameter errors and planet pin diameter errors are of this type. Finally errors such as pitch line run-outs of the sun gear, planets and the internal gear effect the load sharing amongst the planets in a time-varying manner at different levels based on the way they are clocked during the assembly. These errors will be called as time-varying, assembly dependent errors.

Figure 1 illustrates how the positions the profiles of a planet are changed as a function of these three types of manufacturing errors. First, to represent the timeinvariant, assembly dependent errors, apply a certain tooth thickness error $e_{t}$ that moves a given point $A$ on the "perfect" planet flank contacting internal gear to $A^{\prime}$ in the tangential direction by an amount $e_{t} / 2$ while keeping the center of the planet at its nominal position $O$. Next, consider a time-invariant, assembly independent error such as a carrier pinhole position error of magnitude $e_{c}$ to move the center of the planet from $O$ to $O^{\prime \prime}$, as a result, moving point $A^{\prime}$ to $A^{\prime \prime}$ on the tooth flank. Finally, a planet run-out error of magnitude $e_{r}$ (a time-varying and assembly dependent error) is applied to effectively move the center of planet from $O^{\prime \prime}$ to $O^{\prime \prime \prime}$ causing $A^{\prime \prime}$ to move to $A^{\prime \prime \prime}$. The same is true for a point $B$ on the flank that is in contact with the sun gear. The radial and tangential components of the total position error of planet- $i$ in Fig. 1 can be represented by $e_{\text {rad }}^{(i)}$ and $e_{\text {tan }}^{(i)}$ respectively as $(i=1,2, \ldots, n)$

$$
e_{r a d}^{(i)}=e_{c i} \cos \gamma_{c i}+e_{r i} \cos \left(\gamma_{r i}+\theta_{p / c}\right),
$$




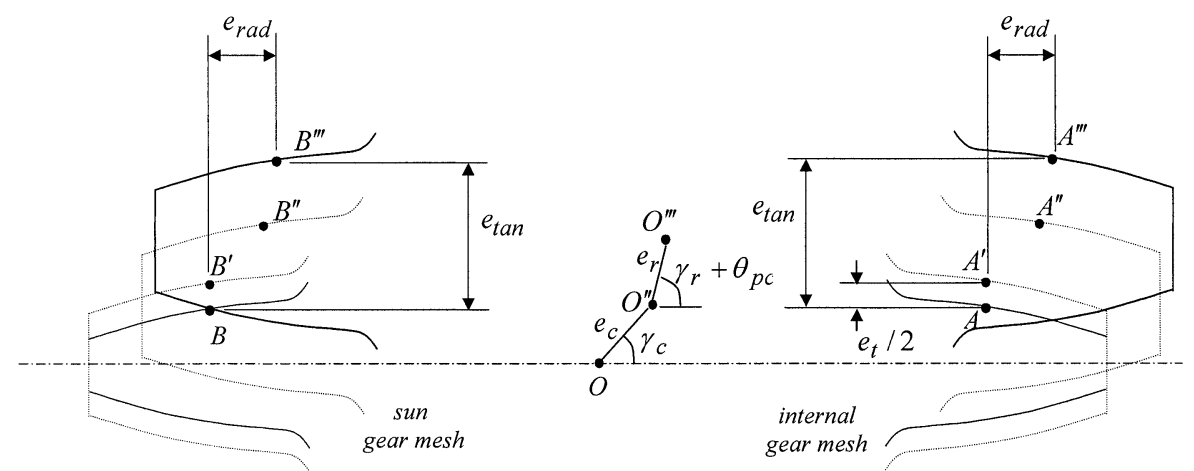

Fig. 1 Influence of different types of manufacturing errors and assembly variations on the position of planet tooth contact surfaces

$$
e_{t a n}^{(i)}=e_{c i} \sin \gamma_{c i}+\frac{e_{t i}}{2}+e_{r i} \sin \left(\gamma_{r i}+\theta_{p / c}\right)
$$

There are a limited number of published studies on planet load sharing. Perhaps to most complete of these studies is a series of papers by Hidaka et al. ${ }^{(2)-(4)}$ who studied the static and dynamic behavior of a three-planet system using lumped mass models. He specifically focused on the motions of floating central members such as the sun gear and the internal gear in the presence of gear run-out errors to align themselves in order to distribute the load equally amongst the three planets. Hidaka showed both experimentally and theoretically that a perfect load sharing in a three-planet gear set is achievable provided that at least one central member is allowed to float confirming other formulations such as Ref.(5). Hidaka and Terauchi $^{(2)}$ also demonstrated that deformations of the carrier and the gears can help improve load sharing amongst the planets. Similar studies by others ${ }^{(6)-(9)}$ reinforce the importance of the gear support (piloting) conditions on planet load sharing. Hayashi et al. ${ }^{(10)}$ performed an experimental study to demonstrate that load sharing improves with the increase in torque.

As above studies mostly focused on three-planet systems, Kahraman ${ }^{(11),(12)}$ focused on planet load sharing characteristics of a four-planet system as such systems are more common in automotive applications. He first used a simplified lumped mass model to study the influence of carrier pin hole and planet run-out errors on planet load sharing under dynamic conditions to state that dynamic load sharing factor is simply a product of a static load sharing factor and a dynamic load factor. Later, Kahraman ${ }^{(12)}$ employed a simplified planet load-sharing model to determine the static planet load sharing of four-planet systems and presented an experimental study for validation of the model predictions.

\subsection{Scope and objectives}

The previous models have major shortcomings originating from the simplicity of the models employed. As the deformations of the gears and the carrier can change the planet load sharing drastically, use of a deformable body contact model like the ones published recently ${ }^{(13),(14)}$ should be more suitable to study load sharing. Secondly, these studies investigated certain aspects of load sharing on a given planetary gear set. Many conclusions reached do not serve as design guidelines as they might change once the configuration is altered by changing design parameters such as number of planets, planet spacing and internal and sun gear mounting conditions.

The main objective of this study develop a deformable body contact mechanics model of a single-stage planetary gear set with $n$ planets to perform a complete study of the planet load sharing under static conditions. Systems with three to six planets will be considered to study the influence of three distinct types of manufacturing errors and assembly variations on planet loads. Piloting conditions of the sun gear and the carrier, and mounting conditions of the internal gear will also be invesitaged in detail. A set of design guidelines representing best practices will be proposed and simplified design formulas will be supplied, whenever possible, for calculating the planets loads as a function of manufacturing errors present.

\section{Computational Model}

There are major difficulties with the use of general purpose, finite element (FE) packages for the gear contact problem resulting often in inaccurate predictions and unacceptably high computational times. The model employed here is designed specifically for the analysis of gear-like contacts eliminating the difficulties associated with conventional FE analysis. Only the most critical features of this model will be summarized here as the details of this model can be found in Refs. (15), (16) and two recent applications ${ }^{(13),(14)}$.

The deformation of the planetary system under load is modeled using a combination of finite element and surface integral techniques. The complex shapes of gears of the planetary system are best modeled by the FE method. However, the contact zones are typically extremely small, and are typically two orders of magnitude smaller than the working depths of the gear teeth requiring a large de- 
gree of freedom concentrated inside the contact zone in order for contact equations to be well conditioned. In addition, the contact zone is not stationary, and moves along the surface of a gear tooth. Therefore, using a pure FE model would require either a mesh that is extremely refined, or a scheme that refines the mesh only in the contact zone and re-meshes for every contact position. Semianalytical deformation models based on the half-space solution for a concentrated load, and numerically integrated over the contact zone do not suffer from the problem of ill conditioning. They can accurately capture the steep displacement gradients inside the contact zone. However, they cannot easily model the shape of the gear teeth. The model used in this study ${ }^{(15)}$ is based on the use the FE models only to compute relative deformation and stresses for points that are away from the contact zones. For points within the contact zone, we use semi-analytical techniques to compute the relative deformations and stresses. The 'near field' semi-analytical solution and the 'far field' finite element solutions are matched at a 'matching surface'.

The FE model implemented here uses separate interpolation schemes for the displacements and coordinates. The tooth surfaces are modeled by elements that have a very large number of 'coordinate nodes', and can therefore accurately represent the involute shape and surface modifications. In the fillet region, the elements have a large number of 'displacement nodes' to correctly capture the steep stress gradients. A hierarchical representation of the system was used, in which the system is built from many substructures, with each substructure in turn being composed of many substructures. The processes of stiffness decomposition and load vector back-substitution makes it possible to keep CPU requirements down to a few seconds per time step while the memory requirements are reduced down significantly.

A Revised Simplex solver is used in this formulation ensures convergence within a predetermined number of iterations unlike the commercial FE packages that use a non-linear equation solver. In the planetary gear system, many rigid body type degrees of freedom or mechanisms are constrained only by the contact conditions often resulting in singular stiffness matrices when a non-linear finite element code with 'gap elements' is used. The approach used here is to attach a reference frame to each individual component, and to carry out the finite element computations for each individual component separately in its own reference frame. As long as each finite element mesh is sufficiently well constrained to its reference frame, the stiffness matrices are well behaved. The Revised Simplex based contact solver can take into account any free mechanisms in the system while computing the contact loads in a very natural manner.

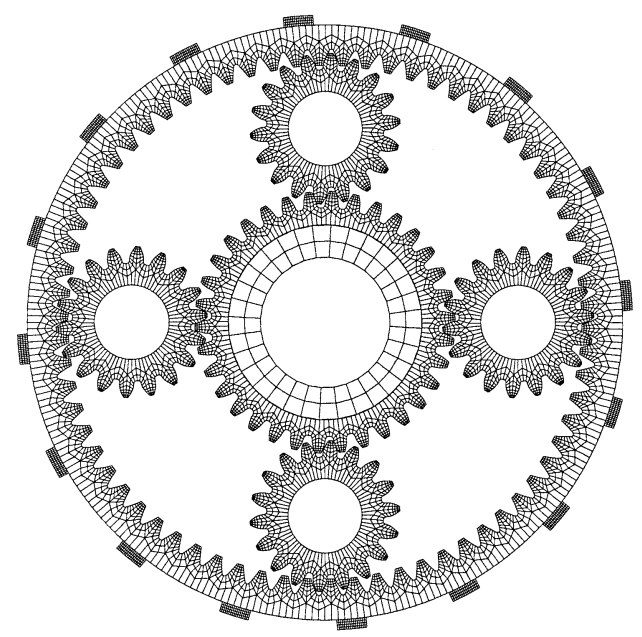

Fig. 2 FE contact mechanics model

Table 1 Parameters of the example system used in this study. All dimensions are in $\mathrm{N}$ and $\mathrm{mm}$ unless specified.

\begin{tabular}{lccc}
\hline & Sun & Planet & Internal \\
\hline Number of teeth & 34 & 18 & 70 \\
Module & 1.5 & 1.5 & 1.5 \\
Pressure angle, deg. & 21.3 & 21.3 & 21.3 \\
Circular tooth thickness & 1.895 & 2.585 & 1.895 \\
Hob tip radius & 0.2 & 0.2 & -- \\
Fillet radius & -- & -- & 0.5 \\
Outer diameter & 52.74 & 30.50 & 119.82 \\
Root diameter & 46.00 & 23.75 & 110.00 \\
Minor diameter & -- & -- & 103.45 \\
Inner (bore) diameter & 26.30 & 15.00 & -- \\
Linear tip modification & 0.01 & 0.01 & -- \\
Tip relief roll angle, deg. & 21 & 27 & -- \\
Young's modulus, $N / \mathrm{mm}^{2}$ & & $2.07 \times 10^{5}$ & \\
Poison's ratio & & 0.3 & \\
Density $\left(\mathrm{kg} / \mathrm{m}^{3}\right.$ ) & & 7800 & \\
\hline
\end{tabular}

\section{Parametric Studies}

\subsection{Example system}

An example planetary gear set whose FE model is shown in Fig. 2 is considered in this study. The design parameters of this system are listed in Table 1. Here, the carrier, the sun gear and the internal gear are the input, output and the reaction members, respectively. An input torque of $T_{i n}=1500 \mathrm{Nm}$ is applied to the system. The baseline configuration in Fig. 2 has a floating sun gear (not supported radially) while the carrier is rigidly supported (piloted). The planetary gear set has four planets $n=4$ and the internal gear is held in a rigid housing by 16 equally spaced straight splines as shown in Fig. 2.

\subsection{Definition of the planet load sharing factor}

The load sharing factor of a given planet- $i, L_{i},(i=1$ to $n$ ), is defined as the ratio of the load carried by this planet to the total load. Different methods of estimating $L_{i}$ have been reported based on gear root stresses ${ }^{(12)}$. Here, planet bearing forces are used instead to determine $L_{i}$. Planet bearing forces $F_{b i}$ at a certain carrier pin hole 


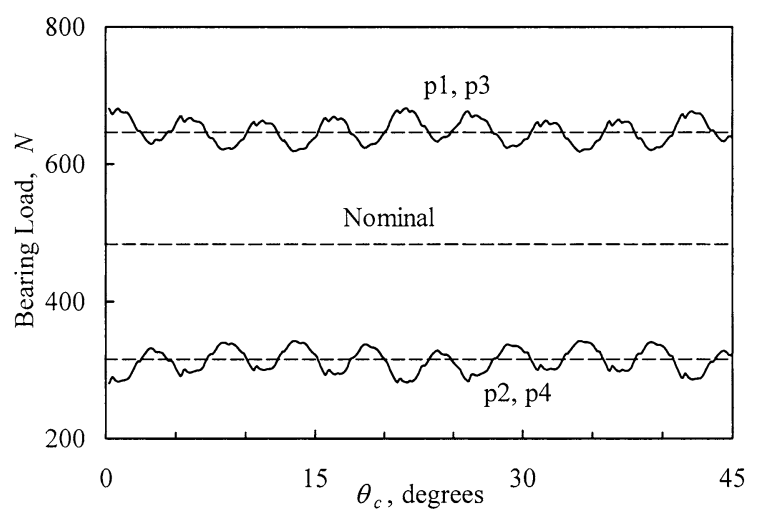

Fig. 3 Planet bearing forces for configuration $\mathrm{A}$ at $T_{\text {in }}=$ $1500 \mathrm{Nm}$

error configuration is shown in Fig. 3. The tangential bearing forces on the bearings of diametrically opposed planets 1 and 3 are equal to each other since the sun gear is allowed to float ${ }^{(11),(12)}$ and fluctuate about an average value of $\bar{F}_{b 1}=\bar{F}_{b 3}$. The same condition is valid for planets 2 and $4, \bar{F}_{b 2}=\bar{F}_{b 4}$. Since at any given rotational angle, the total of the bearing forces is constant, the average value of the load sharing factor of planet- $i, L_{i}$, can be defined as $(i=1$ to $n$ )

$$
\begin{aligned}
& L_{i}=\frac{\bar{F}_{b i}}{F_{b}^{(t o t)}}, \\
& F_{b}^{(t o t)}=\sum_{i=1}^{n} F_{b i}=T_{c} /\left(r_{s}+r_{p}\right)
\end{aligned}
$$

where $n$ is the total number of planets in the gear set and $T_{c}$ is the torque on the carrier (equal to $T_{i n}$ for the example case considered), and $r_{s}$ and $r_{p}$ are the pitch circle radii of the sun gear and the planet gear, respectively. In Fig. 3, $F_{b}^{(t o t)}=1924 \mathrm{~N}, \bar{F}_{b 1}=\bar{F}_{b 3}=648 \mathrm{~N}$ and $\bar{F}_{b 2}=\bar{F}_{b 4}=314 \mathrm{~N}$ resulting in $L_{1}=L_{3}=0.34$ (34 percent) and $L_{2}=L_{4}=0.16$ (16 percent).

\subsection{Influence of manufacturing errors and assem- bly variations}

Carrier planet pin hole position errors are considered first as time-invariant, assembly independent errors. A total $2 n$ parameters fully define the carrier pin hole position errors of an $n$ planet system: magnitudes, $e_{c 1}$ to $e_{c n}$, and position angles, $\gamma_{c 1}$ to $\gamma_{c n}$, where subscript $c$ denotes a carrier pin hole position error. These error parameters are illustrated in Fig. 4 for a four-planet system. A large number of different configurations of pinhole position errors listed as configurations $A$ to $F$ in Table 2 with $\left|e_{c i}\right| \leq 50 \mu \mathrm{m}$ are applied to the example planetary gear set. These configurations $A$ to $F$ defined in Table 2 involve only the tangential pin hole position errors with $\gamma_{c i}=\pi / 2$ or $3 \pi / 2$ resulting in $e_{\text {rad }}^{(i)}=0$ as the investigation of errors in radial direction showed that their impact is negligible.

The first configuration in Table 2 is formed by introducing a pinhole position error of magnitude up to $50 \mu \mathrm{m}$ on planet 1 alone in tangential direction while all

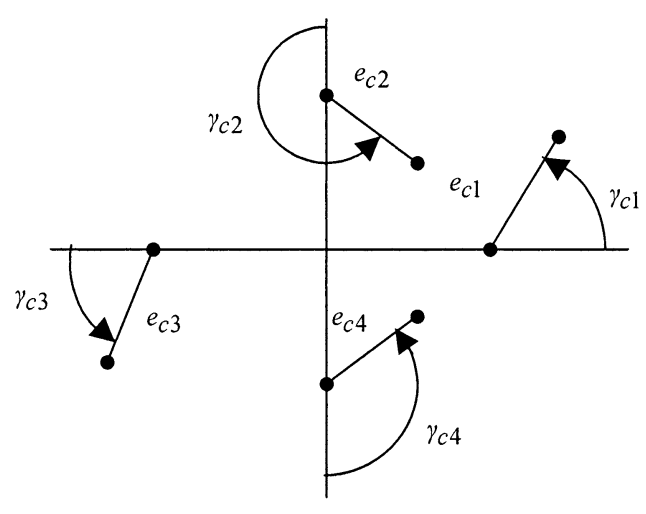

Fig. 4 Graphical representation of carrier pinhole errors of a 4-planet system

Table 2 Different configurations of manufacturing errors con-

\begin{tabular}{|c|c|c|}
\hline $\begin{array}{l}\text { Configurati } \\
\text { on }\end{array}$ & Mathematical Representation & $\begin{array}{c}\text { Graphical } \\
\text { Representation }\end{array}$ \\
\hline $\mathrm{A}$ & $\begin{array}{r}e_{c i}=0, i=2 \text { to } 4 \\
-50 \leq e_{c 1} \leq 50 \mu m\end{array}$ & 9 \\
\hline B & $\begin{array}{c}e_{c 1}=-e_{c i} \quad i=2 \text { and } 4 \\
-50 \leq e_{c 1} \leq 50 \mu m\end{array}$ & 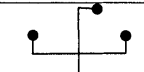 \\
\hline C & $\begin{array}{c}e_{c 1}=e_{c 2} \\
e_{c i}=0, i=3 \text { and } 4 \\
-50 \leq e_{c 1} \leq 50 \mu \mathrm{m}\end{array}$ & ๑ \\
\hline D & $\begin{array}{c}e_{c 1}=-e_{c 2} \\
e_{c i}=0, i=3 \text { and } 4 \\
-50 \leq e_{c 1} \leq 50 \mu \mathrm{m}\end{array}$ & 9 \\
\hline $\mathrm{E}$ & $\begin{array}{c}e_{c 1}=e_{c 3}, e_{c 1}=-e_{c i} \\
e_{c i}=0, i=2 \text { and } 4 \\
-50 \leq e_{c 1} \leq 50 \mu \mathrm{m}\end{array}$ & 9 \\
\hline F & $\begin{array}{c}e_{c 1}=e_{c 3}, \quad i=2 \text { and } 4 \\
-50 \leq e_{c 1} \leq 50 \mu m\end{array}$ & • \\
\hline G & $\begin{array}{c}e_{t i}=0, i=2 \text { to } 4 \\
-30 \leq e_{t 1} \leq 30 \mu m\end{array}$ & $\theta+0$ \\
\hline $\mathrm{H}$ & $\begin{array}{c}e_{t 1}=-e_{t 2} \\
e_{t i}=0, i=3 \text { and } 4 \\
-30 \leq e_{t 1} \leq 30 \mu m\end{array}$ & 9 \\
\hline $\mathrm{J}$ & $\begin{array}{c}e_{t 1}=e_{t 3}, e_{t 2}=e_{t 4}=0 \\
-30 \leq e_{t 1} \leq 30 \mu \mathrm{m}\end{array}$ & 6 \\
\hline $\mathrm{K}$ & $\begin{array}{c}e_{t 1}=e_{t 3}, e_{t 2}=e_{t 4} \\
e_{t 1}=-e_{t 2} \\
-30 \leq e_{t 1} \leq 30 \mu \mathrm{m}\end{array}$ & $\theta$ \\
\hline $\mathrm{L}$ & $\begin{array}{c}e_{t 2}=e_{t 3}, e_{t 1}=e_{t 4} \\
e_{t 2}=-e_{t 1} \\
-30 \leq e_{t 1} \leq 30 \mu \mathrm{m}\end{array}$ & 9 \\
\hline$M$ & $\begin{array}{l}0 \leq e_{r 1} \leq 25 \mu m \\
\text { other } e_{r i}=0\end{array}$ & $\bullet$ \\
\hline $\mathrm{N}$ & $\begin{array}{c}e_{r 1}=e_{r 3} \\
0 \leq e_{r 1} \leq 25 \mu m, \text { other } \quad e_{r i}=0\end{array}$ & 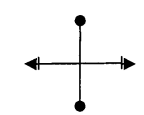 \\
\hline $\mathrm{P}$ & $\begin{array}{c}e_{r i}=20 \mu m, i=1 \text { to } 4 \\
\gamma_{r 1}=\gamma_{r 2}, \gamma_{r 3}=\gamma_{r 4}, \quad \gamma_{r 3}=\pi+\gamma_{r 1}\end{array}$ & $\mapsto \underbrace{}_{1}$ \\
\hline $\mathrm{Q}$ & $\begin{array}{c}e_{r i}=20 \mu m, i=1 \text { to } 4 \\
\gamma_{r 1}=\gamma_{r 3}, \quad \gamma_{r 2}=\gamma_{r 4}, \quad \gamma_{r 2}=\pi+\gamma_{r 1}\end{array}$ & $+\sum_{1}^{5}$ \\
\hline
\end{tabular}
sidered in this study. 


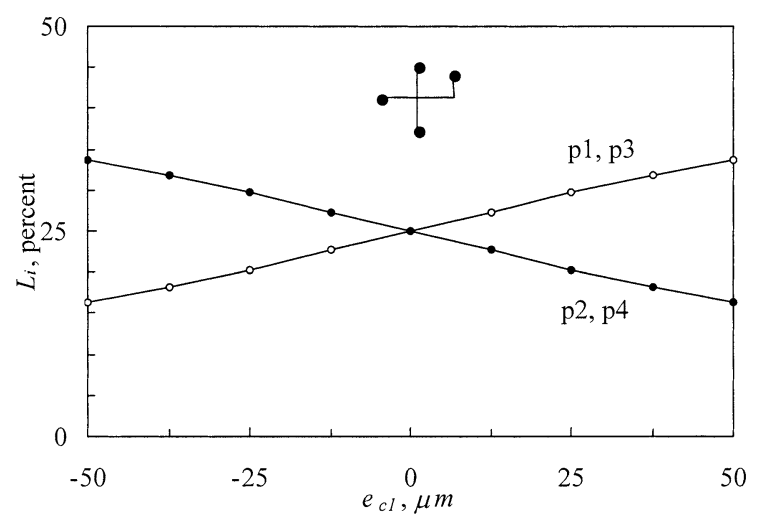

Fig. 5 Variation of $L_{i}$ with $e_{c 1}$ for configuration $A$

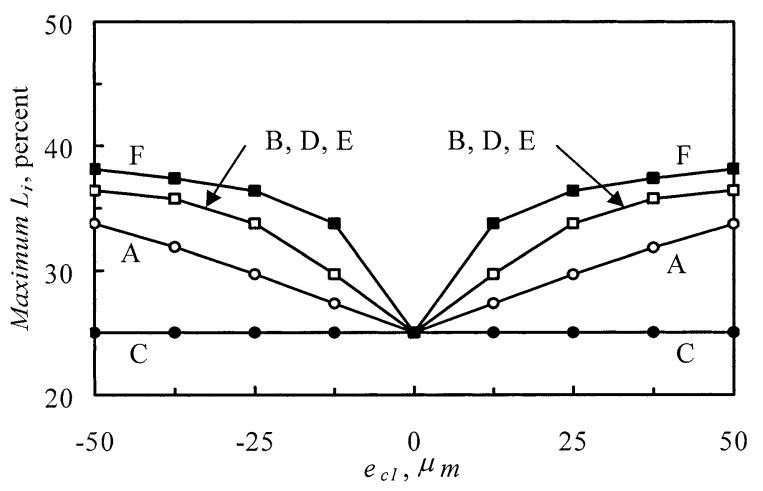

Fig. 6 Variation of maximum $L_{i}$ with $e_{c 1}$ for configurations $A$ to $F$

other planet pinholes are at their nominal positions, i.e. $e_{c 2}=e_{c 3}=e_{c 4}=0$. It is also assumed that no other manufacturing errors are present. As shown in Fig. 5 for configuration $A$ that the load on planet 1 decreases linearly as $e_{c 1}$ is reduced. Planet pairs $\mathrm{p} 1 / \mathrm{p} 3$ and $\mathrm{p} 2 / \mathrm{p} 4$ carry equal loads primarily due to floating sun gear. Here the value of $L_{1}=L_{3}$ is as large as 34 percent for $e_{c 1}=50 \mu \mathrm{m}$ and as low as 16 percent for $e_{c 1}=-50 \mu \mathrm{m}$. Meanwhile for $e_{c 1}=50 \mu \mathrm{m}$, planets 1 and 3 experience higher stresses than planets 2 and 4 .

In Fig. 6, maximum values of $L_{i}$ for configurations $A$ to $F$ in Table 2 are plotted against $e_{c 1}$. Configuration $B$ in Table 2 is similar to Configuration $A$ except $e_{c 1}=-e_{c i}$, $i=2$ to 4 , suggesting that effective $e_{c 1}$ is now twice as in configuration $A$. In Fig. 6, maximum $L_{i}$ values for configuration $B$ are higher than those of Configuration $A$. At $e_{c 1}=50 \mu \mathrm{m}$, maximum $L_{i}$ is around 36 percent. In configuration $C$ with $e_{c 1}=e_{c 2}, \gamma_{c 1}=\gamma_{c 2}$ and $e_{c 3}=e_{c 4}=0$, the load sharing is perfect. Configurations $D$ with $e_{c 2}=-e_{c 1}$ and $E$ with $e_{c 1}=e_{c 3}$ and $\gamma_{c 1}=\gamma_{c 3}$ yield exactly the same results as Configuration $B$. The worst case amongst configurations $A$ to $F$ in Table 2 is found to be Configuration $F$ with $e_{c 1}=e_{c 3}=-e_{c 2}=-e_{c 4}$. In Fig. 6, maximum $L_{1}=L_{3}$ increases to 38 percent for $e_{c 1}=50 \mu \mathrm{m}$ indicating that planets 2 and 4 each carry about 12 percent of the total load that is less than half of the ideal planet load.

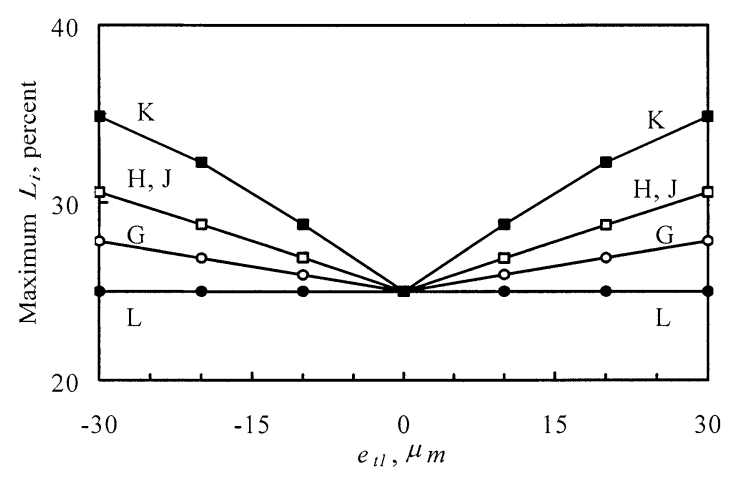

Fig. 7 Variation of maximum $L_{i}$ with $e_{t 1}$ for configurations $G$ to $L$

Secondly, average planet tooth thickness errors (timeinvariant, assembly-dependent) considered where $e_{t i}$ is the deviation of the average tooth thickness from the nominal value. A positive $e_{t i}$ implies that the planet tooth is thicker than its nominal value. For an $n$-planet system, $n$ parameters are sufficient to define the overall error configuration. A number of different configurations $G$ to $L$ shown in Table 2 are considered here as planets with a tooth thickness larger than the nominal tooth thickness are depicted by larger circles and visa versa. For instance, the results for configurations $G$ to $L$ are compared in Fig. 7 within the range $e_{t 1}$ within -30 to $30 \mu \mathrm{m}$. Configuration $K$ represents the tooth thickness arrangement yielding the worst load sharing condition, as two diametrically opposed planets are the largest while the other two are the smallest. It is worth noting that configuration $K$ with $\left|e_{t i}\right|=30 \mu \mathrm{m}$ results in the same maximum $L_{i}$ values (35 percent) for configuration $F$ in Fig. 6 with $e_{c 1}=15 \mu \mathrm{m}$ demonstrating that $e_{t i}$ influence the load sharing the same way as $e_{c i}$ in accordance with Eq. (1).

One last observation from Table 2 and Fig. 7 can be made to demonstrate assembly dependence of the tooth thickness variations. Configuration $L$ in Table 2 is obtained from configuration $K$ (the worst case) by simply switching the positions of two adjacent planets resulting in $e_{t 1}=e_{t 2}=-e_{t 3}=-e_{t 4}$. The resultant change in load sharing is rather phenomenal as the worst case of load sharing is reduced to an ideal load sharing as shown in Fig. 7. This suggests that given $n$ planets with certain $e_{t i}$ can cause significantly different planet loads depending on the sequence they are assembled in a planetary gear set.

Planet run-out errors in configurations $M$ to $P$ in Table 2 are considered to demonstrate the influence of timevarying and assembly dependent errors. Here, the high point of the run-out of a planet rotates with the planet as its component in the tangential direction given by Eq. (1) varies harmonically making it a rotation (time) dependent error. A total of $2 n$ parameters define completely the planet run-out errors on an $n$-planet system, magnitudes, $e_{r i}$, and the initial orientation angle of high point, $\gamma_{r i}$, de- 


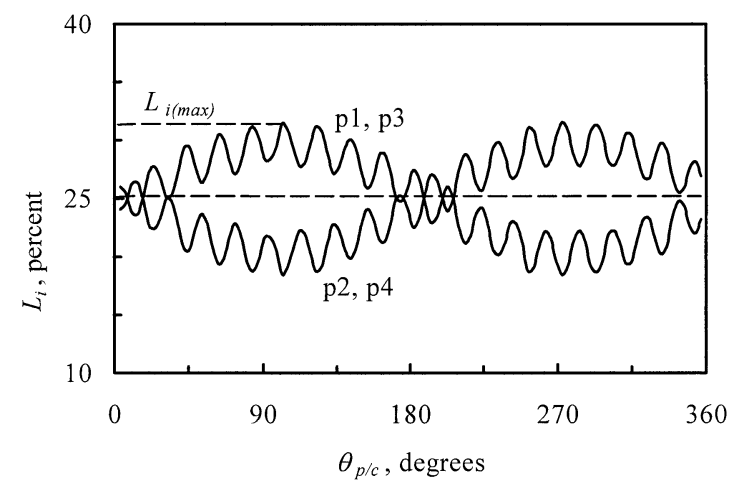

Fig. 8 Variation of $L_{i}$ with $e_{r 1}$ for configuration $M$ at $T_{i n}=$ $1500 \mathrm{Nm}$

fined positive in counter clockwise direction from the outward radial axis as shown in Fig. 4. Once a planetary gear set is assembled the relative orientations $\gamma_{r i}$ are fixed, yet given a set of $n$ planets with given $e_{r i}$ can be clocked in any arbitrary $\gamma_{r i}$ in assembly making these errors assembly dependent as well.

Figure 8 shows the variation of maximum $L_{i}$ with rotation angle of the planets relative to carrier $\theta_{p / c}$ for configuration $M$ in Table 2 for $e_{r 1}=25 \mu \mathrm{m}$ with other $e_{r i}=0$. Given an initial position $\gamma_{r i}=0, e_{t a n}^{(i)}=0$ in Eq. (1) at $\theta_{p / c}=0$ resulting in $L_{i}=25$ percent. As the pinions rotate, planets 1 and 3 carry larger share of the load reaching their maximum value of nearly 32 percent at $\theta_{p / c}=\frac{\pi}{2}$. This is the position at which the high point of the run-out of planet 1 is in the tangential direction and $e_{t a n}^{(1)}$ is maximum. At $\theta_{p / c}=\pi$ and $2 \pi$, again all $L_{i}=25$ percent since $e_{\text {tan }}^{(1)}=0$, and at $\theta_{p / c}=3 \pi / 2, L_{1}=L_{3}$ are minimum. The curve for $L_{2}=L_{4}$ follows the same exact shape except it is the mirror image of the curve for $L_{1}=L_{3}$ about the ideal load sharing line at 25 percent. The same load sharing trends are observed for configuration $N$, only the load sharing is more severe due to the fact that planets 1 and 3, both have the same amount of run out error in the same direction. The maximum $L_{i}$ values plotted in Fig. 9 as a function of $e_{r i}$ indicate that maximum $L_{i}$ is as high as 32 and 35 percent for configurations $M$ and $N$, respectively.

Configuration $P$ in Table 2 is obtained by applying the same amount of run-out error to each planet $e_{r i}=20 \mu \mathrm{m}$, $i=1$ to 4 at initial angles $\gamma_{r 1}=\gamma_{r 2}=\gamma_{r 3}+\pi=\gamma_{r 4}+\pi$. In this case, all $L_{i}$ remain at 25 percent. This perfect load sharing behavior changes significantly when the same system is reassembled to change the initial positions of planets 2 and 3 as shown in Table 2 to obtain configuration $Q$. Here, the maximum value of $L_{i}$ is about 36 percent representing the worst assembly configuration of planet run-out errors.

Different cases are analyzed for sun gear and the internal gear run out errors were also considered to show that for a case with at least one of the central members floating, run-out errors of central member (sun gear, carrier and internal gear) have very limited influence on the

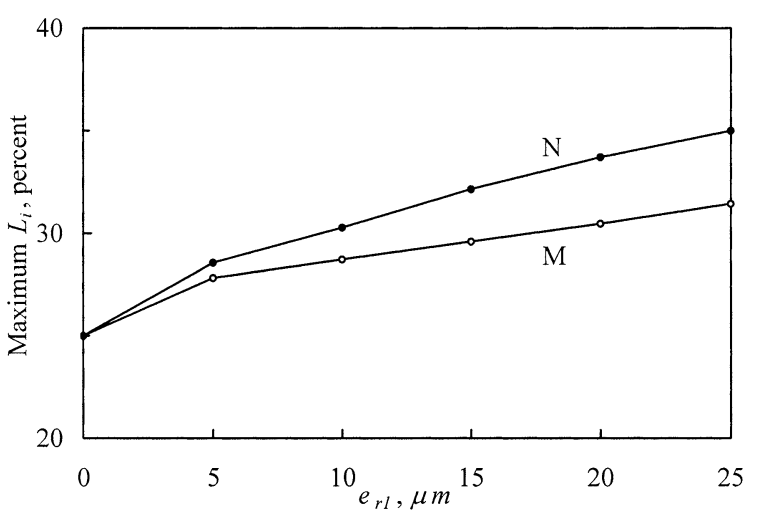

Fig. 9 Variation of maximum $L_{i}$ with $e_{r 1}$ for configurations $M$ and $N$

way the load distributed to the planets.

\subsection{Influence of piloting conditions on planet load sharing}

The effect of sun gear and carrier piloting conditions and internal gear mounting conditions on the planet load sharing characteristics is investigated here. It was reported previously that the load sharing characteristics could change drastically when the piloting conditions are varied $^{(3),(4),(17)}$. A sun gear or the carrier that is allowed to float radially has the ability to move to a new center to improve on load sharing amongst the planets. Here, given the present splined internal gear mounting condition of the example four-planet system, following four piloting conditions are possible: (1) floating sun and carrier $(F F),(2)$ floating sun and piloted carrier $(F P),(3)$ piloted sun and floating carrier $(P F)$, and (4) piloted sun gear and carrier $(P P)$.

All of the results presented up to this point correspond to the piloting condition $F P$ condition. Cases $F P, P F$ and $F F$ are achieved by removing the bearing constraint from that respective member while a piloted condition is obtained by a support stiffness matrix representing a typical rolling element bearing. In Fig. 10 for configuration A of Table 2, the same results are obtained for when at least one of the two members is allowed to float (cases $F F, P F$ and $F P$ ). When both central members are piloted (case PP), the load sharing is the poorest as there is no room for any central member to adjust its radial position to distribute the total load equally amongst the planets. For instance, for $e_{c 1}=50 \mu \mathrm{m}$, the maximum load on planet 1 is nearly 47 percent. The loads on the diametrically opposed planets are no longer equal, $L_{1} \neq L_{3}$ and $L_{2} \neq L_{4}$. For the same error, floating at least one member reduces the value of $L_{1}$ to 34 percent and diametrically opposed planets carry the same amount of load. This suggests that piloting both, the sun gear and carrier, could not be an acceptable design practice. At least one central member must be allowed to float radially. 


\subsection{Influence of number of planets}

The example equally spaced, four-planet system shown in Fig. 2 is modified first to obtain 3, 5 and 6 planet versions of the same system. The spacing of the planets are kept as uniform as possible, as allowed by the least mesh angle. The same input torque value as before is maintained in this analysis with a floating sun gear, a splined internal gear and the same error configuration $A$ from Table 2. The maximum $L_{i}$ values are compared for the systems with $n=3,4,5$ and 6 in Fig. 11. First of all, the three planet system has a constant $L_{i}=33.3$ percent, $i=1$ to 3 , independent of $e_{c 1}$ indicating that load sharing amongst the planets is not an issue for a three planet system with at least one floating central member. For $n=4$, the maximum $L_{i}$ values are consistently less than those for $n=3$. The difference is the largest when there is no error in the system $\left(L_{i, n=4} / L_{i, n=3}=3 / 4\right)$ while this advantage of the four-planet system erodes significantly as $e_{c 1}$ is increased. For instance, at $e_{c 1}=50 \mu \mathrm{m}, L_{i, n=4} / L_{i, n=3}=1.02$ suggesting that the maximum planet load on the four-planet system is larger that that of a three-planet counterpart.

Similar behavior is evident for $n=5$ and $n=6$ as well. For $n=6, L_{i, n=6} / L_{i, n=3}=0.5$ when there is no error, mean-

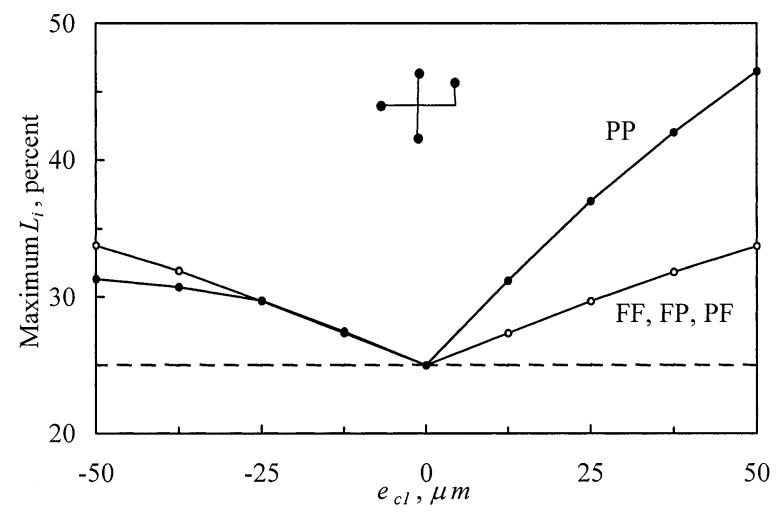

Fig. 10 Variation of maximum $L_{i}$ with $e_{c 1}$ for configuration $A$ and piloting conditions FF, FP, PF and PP

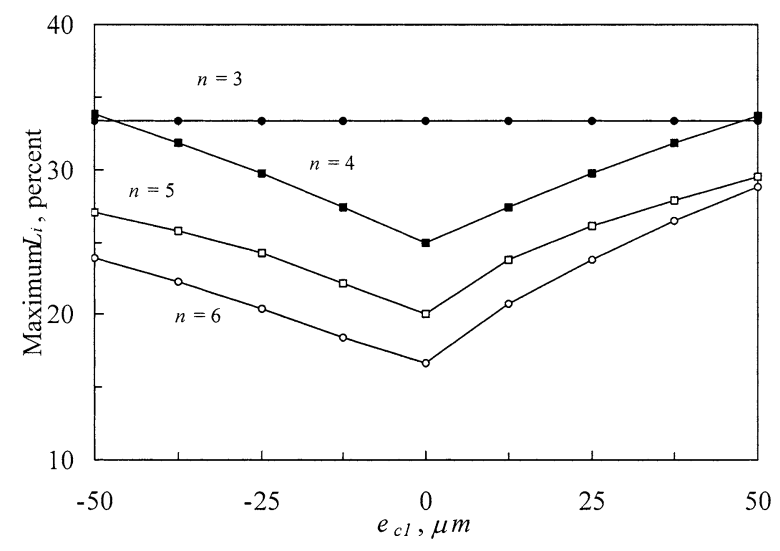

Fig. 11 Variation of maximum $L_{i}$ with $e_{c 1}$ for systems having different number of planets $n$ ing that the maximum planet load is reduced to half by doubling the number of planets. When $e_{c 1}=50 \mu \mathrm{m}$, however, $L_{i, n=6} / L_{i, n=3}$ is only 0.87 suggesting that the reduction in maximum planet load obtained by doubling $n$ to 6 is only 13 percent. Figure 11 shows clearly that the load sharing gets worse and the impact of manufacturing errors on planet loads becomes more important as $n$ is increased.

\section{Discussion of Results}

Results presented here state that not only the manufacturing errors and assembly variations but also a number of system level planetary gear set design parameters influence the load carried by each individual planet. Especially, the load sharing characteristics are uniquely different for systems with different number of planets. For $n=3$, perfect load sharing conditions are ensured regardless of type or magnitude of error, provided that one central member is floating. For $n=4$ with a floating central member, diagonally opposed members carry equal amounts of load while differences between two adjacent planet loads is strongly dependent on manufacturing errors and assembly variations. For systems with larger number of planets $(n=5$ or $n=6$ ), the total load is shared rather poorly wiping out most of the expected reductions in planet loads especially when the gear or carrier errors are excessive.

While the load sharing mechanism is quite complex for $n \geq 5$, there exists a simple relationship for any system with $n=4$. This relationship that is arrived heuristically states that manufacturing errors acting on each planet can be used to define an effective planetary error parameter $E$

$$
E=\left|\left(e_{\text {tan }}^{(1)}+e_{\text {tan }}^{(3)}\right)-\left(e_{\text {tan }}^{(2)}+e_{\text {tan }}^{(4)}\right)\right| \quad(\text { for } n=4 \text { only) }
$$

that alone defines maximum $L_{i}$. In order to test the above hypothesis, $E$ is calculated for each error configuration in Table 2 and plotted against $L_{i}$ as shown in Fig. 12. Here, regardless of the type of the error and configuration at which it is applied, $L_{i}$ is defined by the corresponding $E$ value. This way, a design curve like Fig. 12

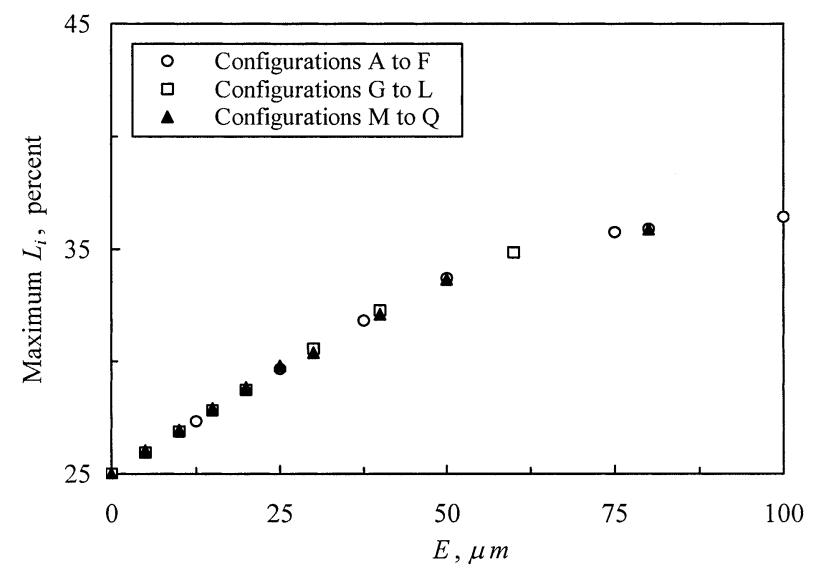

Fig. 12 Variation of maximum $L_{i}$ with effective error $E$ for a system having four planets 
can be constructed by analyzing the same four-planet system under a small number of error conditions representing different levels of $E$. This curve can then be used as a design curve to determine the maximum $L_{i}$ from which the maximum excess planet load can be determined as $T_{\text {excess }}=\left(L_{i}-\frac{1}{4}\right) T_{\text {in }}$.

\section{Conclusions}

In this paper, a state-of-the-art contact mechanics model of a planetary gear has been employed to study the effect of a number of manufacturing and assembly related carrier and gear errors on the load sharing amongst the planets. Three different groups of errors considered are: (i) time-invariant, assembly-independent errors such as carrier planet pinhole position errors, (ii) time-invariant, assembly-dependent errors such as planet tooth thickness errors, and (iii) time-varying, assembly-dependent errors such as gear run-out errors. With such errors present, planet load sharing characteristics of an $n$-planet system ( $n=3$ to 6 ) have been investigated for different piloting configurations under static conditions. Finally, the load sharing behavior as a function of key manufacturing errors and design parameters has been quantified including design formulas for better planet load sharing behavior.

\section{Acknowledgements}

We thank Borg-Warner Automotive for sponsoring this research study. We also acknowledge Dr. S. Vijayakar of Advanced Numerical Solutions, Inc. for making available a gear contact analysis program 2DPLANETARY.

\section{References}

(1) Balasubramanian, B., Dynamische Lastverteilung in Planetensantzen, Ph. D. Dissertation, Universitat Karlsruhe, Germany, (1983).

( 2 ) Hidaka, T. and Terauchi, Y., Dynamic Behavior of Planetary Gear-1st Report, Load Distribution in Planetary Gear, Bulletin of the JSME, Vol.19 (1976), pp.690-698.

( 3 ) Hidaka, T., Terauchi, Y. and Dohi, K., On the Relation between the Run out Errors and the Motion of the Center of Sun Gear in a Stoeckicht Planetary Gear, Bulletin of the JSME, Vol.22 (1979), p.167.
( 4 ) Hidaka, T., Terauchi, Y. and Nagamura, K., Dynanic Behavior of Planetary Gear-7th Report, Influence of the Thickness of Ring Gear, Bulletin of the JSME, Vol.22 (1979), p.170.

( 5 ) Muller, H.W., Epicyclic Drive Trains, (1982), Wayne State University Press, Detroit.

( 6 ) Seager, D.L., Load Sharing among Planet Gears, SAE Paper 700178, (1970).

( 7 ) Kasuba, R. and August, R., Torsional Vibrations and Dynamic Loads in a Basic Planetary Gear System, Transactions of the ASME, Vol.108 (1986), pp.348353.

( 8 ) Ma, P. and Botman, M., Load Sharing in a Planetary Gear Stage in the Presence of Gear Errors and Misalignments, Transactions of the ASME, Vol.107 (1985), 84-DET-54.

(9) Jarchow, F., Development Status of Epicyclic Gears, International Power Transmission and Gearing Conference, Chicago, (1989).

(10) Hayashi, T., Li, Y., Hayashi, I., Endou, K. and Watanabe, W., Measurement and Some Discussions on Dynamic Load Sharing in Planetary Gears, Bulletin of the JSME, Vol.29 (1986), pp.2290-2297.

(11) Kahraman, A., Load Sharing Characteristics of Planetary Transmissions, Mechanisms and Machine Theory, Vol.29, No.8 (1994), pp.1151-1165.

(12) Kahraman, A., Static Load Sharing Characteristics of Transmission Planetary Gear Sets: Model and Experiment, SAE Paper 1999-01-1050, (1999).

(13) Kahraman, A. and Vijaykar, S., Effect of Internal Gear Flexibility on the Quasi-Static Behavior of a Planetary Gear Set, ASME Journal of Mechanical Design, Vol.123 (2001), pp.408-415.

(14) Kahraman, A., Kharazi, A.A. and Umrani, M., A Deformable Body Dynamic Analysis of Planetary Gears with Thin Rims, Journal of Sound and Vibration, Vol.262 (2003), pp.752-768.

(15) Vijayakar, S., A Combined Surface Integral and Finite Element Solution for a Three-Dimensional Contact Problem, International Journal for Numerical Methods in Engineering, Vol.31 (1991), pp.525-545.

(16) Vijayakar, S., Busby, S. and Houser, D.R., Linearization of Multibody Frictional Contact Problems, Computers and Structures, Vol.29 (1987), pp.569-576.

(17) Dizioglu, B., Kinematik des Lastausgleiches in Planetengetrieben, VDI-Berichte, Nr.167 (1971), pp.193198. 